\title{
Microplastics in The Bali Strait : Comparison of Two Sampling Methods
}

\author{
Defri Yona ${ }^{1,2 *}$, Zefanya Nandaningtyas ${ }^{1}$, Bernads Daniel Marolop Siagian ${ }^{1}$, Syarifah Hikmah \\ Julinda Sari ${ }^{1,2}$, Agung Yunanto ${ }^{3}$, Feni Iranawati ${ }^{1,2}$, Mochamad Arif Zainul Fuad ${ }^{1,2}$, Junika Chintia \\ Ayu Putri², Mela Dita Maharani²
}

\author{
${ }^{1}$ Marine Science Department, Fisheries and Marine Sciences Faculty, Brawijaya University \\ ${ }_{2}^{2}$ Marine Resources Exploration and Management Research Group, Fisheries and Marine Sciences Faculty, \\ Brawijaya University \\ Jl. Veteran, Malang, East Java, 65151,Indonesia \\ 3 Institute for Marine Research and Observation \\ Jl. Baru Perancak Negara, Bali Indonesia \\ Email : defri.yona@ub.ac.id
}

\begin{abstract}
Two methods of microplastics sampling in the Bali Strait, manta net (250 $\mu \mathrm{m}$ mesh size) and plankton net (20 $\mu \mathrm{m}$ mesh size), were compared. The difference in the mesh sizes could result in the difference of the microplastics found. Water samples from both sampling tools were analyzed with filtration and all organic materials were removed using Hydrogen Peroxide. Natrium chloride $(\mathrm{NaCl})$ was used to further separate microplastics and organic materials based on its density. The result identified three types of microplastics found in Bali Strait: fibers, films and fragments with total abundances of microplastics were $32.48 \times 10^{2}$ particles.m $\mathrm{m}^{-3}$ and $16.33 \times 10^{2}$ particles. $\mathrm{m}^{-3}$ using manta net and plankton net, respectively. These results indicated that the numbers of microplastics per cubic metres was higher using manta net sampling tool compared to plankton net. This may likely caused by the smaller size of the mesh used and also the sampling area covered using manta net.
\end{abstract}

Keywords: microplastics, marine debris, manta net, plankton net, Bali Strait

\section{Introduction}

Waste pollution has become a main problem all around the world as human population has also increasing. One type of wastes which has become serious problem and located in both land and marine environments is plastic. Plastic has remained one of the most used materials in the household and ranging to the industrial business. For 75 years, the amount of plastic usage has increased dramatically from 1.5 million tons to 322 million tons a year and it is estimated that as much as 4-12 million tons of plastic located in the sea are from the land (Coppock et al., 2017).

Marine debris is defined as solid material which produced by human activities and accidentally or purposely left in a marine environment (National Oceanic and Atmospheric Administration, 2013). Marine debris in the sea is divided into four groups based on its size: megaplastics ( $>1 \mathrm{~m})$, macroplastics $(<1 \mathrm{~m})$, mesoplastics $(<2.5 \mathrm{~cm})$ and microplastics $(<5$ $\mathrm{mm}$ ) (GESAMP, 2016). Marine debris will eventually go through a decomposing process which will result in the debris turning into microplastics and nanoplastics by many physical, chemical, and biological processes.
The term 'microplastic' refers to a plastic particle which size is smaller than $5 \mathrm{~mm}$ (ranging from millimetre to micrometre) with variations regarding the colour and the shape (ranging from small fragment to long fibre-like strands) (Kovač Viršek et al., 2016). The spread of microplastic is vast starting from the shore lines to the deepest oceans (Coppock et al., 2017). Microplastic is divided into two categories which are primary and secondary microplastics. Primary microplastics are plastic particles that are intentionally made with the purpose of being used in the cosmetics and chemicals industry which could end up in the ocean (GESAMP, 2016). It is generally use for cleaning and cosmetic products, or pellets from animal feed and powdered resin (Gregory, 1996). Secondary microplastic is formed from the degradation process of plastic affected by light, temperature, and organisms (GESAMP, 2016; Kovač Viršek et al., 2016). It is commonly found in aquatic environment and is generally a fraction from bigger plastic waste and clothing fiber (Kovač Viršek et al., 2016).

Based on its visual appearance, microplastics are divided into three categories: fragments, films and 
fiber. Fragments are fractions from plastic products which are thick, stiff and have an inconsistent shape ( Hidalgo-Ruz et al., 2012a; Hastuti et al., 2014; Kovač Viršek et al., 2016). According to Hidalgo-Ruz et al. (2012), plastic particles which are thin, flexible and generally transparent are categorized as film. Plastic fiber which are originally part of fishing nets, rope and cloth fiber. Kovač Viršek et al., (2016) classifies microplastic into 3 additional categories besides fragment, film, and fiber, which are pellets, granules and foam. Pellets and granules have the same visual trait which generally is a ball-like shape but inconsistent and with a variety of color. The main distinguishment between pellets and granules is that granules have a diameter as big as $1 \mathrm{~mm}$ while pellets have a diameter of $5 \mathrm{~mm}$ or more. The last category is foam, where foam generally comes from styrofoam which traits such as being soft, no consistent shape and a whitish ot yellowish hue.

The purposes of this study were to identify the types and total number of microplastics and to compare the abundance of microplastics obtained from two different sampling tools (manta net and plankton net).

\section{Material and methods}

This research was conducted in the Bali Strait area in October 2017 (Figure 1.). Water samples were acquired from two separate sites located about 3 miles from the coastline. The first sampling site was located near fishing port and the second one was located near estuary. Water samples were taken using manta net (Figure 2a.) and plankton net (Figure $2 b$.) in both sampling sites. Both sampling tools have been used in many studies. Manta net has a mesh size of $250 \mu \mathrm{m}$ with a mouth size of $59 \mathrm{~cm}$ (length) $\mathrm{x}$ $25 \mathrm{~cm}$ (width). Manta net was pulled by boat with a speed of 1 knots for about 10 mins depending on the physical condition of the study areas (waves, wind and currents) (Kovač Viršek et al., 2016).

Plankton net sampling method was conducted with a mesh size of $20 \mu \mathrm{m}$. About $15 \mathrm{~L}$ of seawater was taken using a basket and then filtered using plankton net. About $250 \mathrm{ml}$ filtered seawater from both manta net and plankton net were then placed in polyethylene bottle sample and kept cool until further analysis in the laboratory. To reduce plastic contamination, all the plastic equipment used in the sampling process were cleaned and rinse with the distilled water at least three times before used. Also, blank samples were taken and filled with filtered seawater to evaluate the contamination on-board. These procedures were conducted according to the standardized protocol for monitoring microplastics in seawater (Gago et al., 2018).

\section{Microplastic analysis}

In the laboratory, water samples were analyzed to identify the type of microplastics and also its abundances. There are several steps in the analyzing and identifying microplastics: filtering, wet peroxide oxidation (WPO) process, density separator and

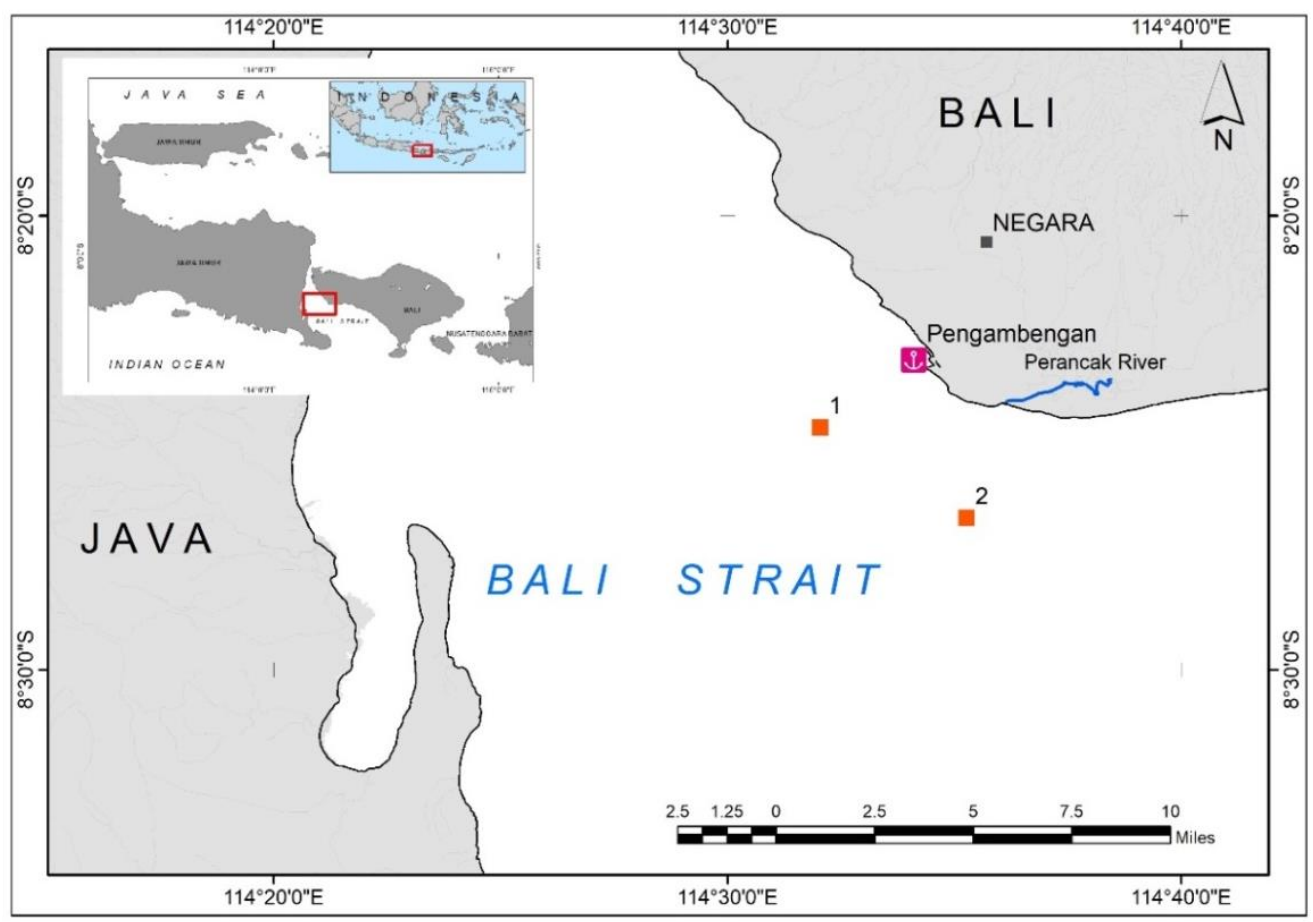

Figure 1. Water Sampling Locations in Bali Strait 


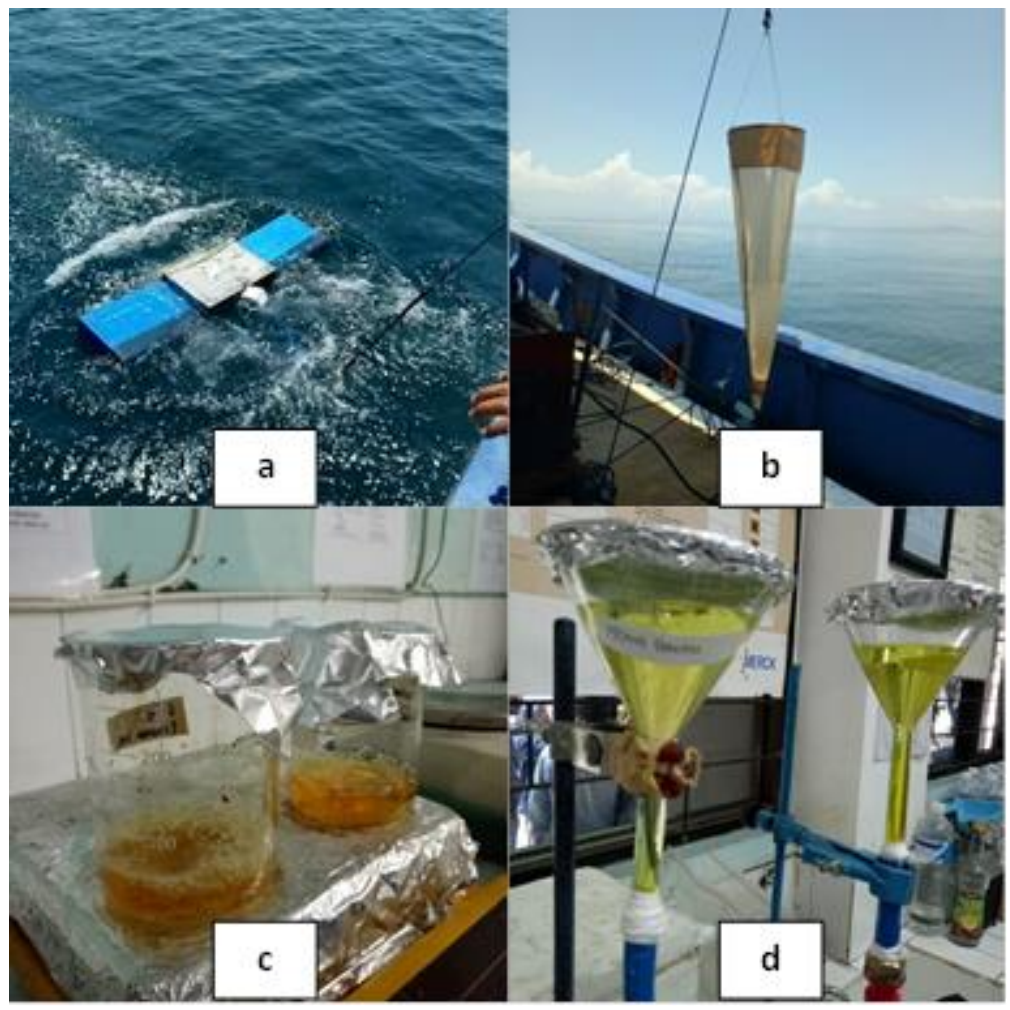

Figure 2. Microplastics sampling using manta net (a) and plankton net (b), Wet Peroxide Oxidation (WPO) process (c) and microplastics isolation process using density separator $(d)$

gravimetric analysis. This method was used to identify microplastics size with the range from $5 \mathrm{~mm}$ to 0.33 $\mathrm{mm}$ (Misura et al., 2015).

Microplastic samples were filtered using $5 \mathrm{~mm}$ and $0.3 \mathrm{~mm}$ filter with the assumption that samples that could pass the $5 \mathrm{~mm}$ filer were considered as microplastics and particles that do not pass were counted as macroplastics. After the filtration process, a beaker glass filled with microplastics particles was then heated in an oven with a temperature of $90^{\circ} \mathrm{C}$ for 24 hours or longer until the sample was dry (Misura et al., 2015). Dried samples were then separated from the organic materials using wet peroxide oxidation (WPO) process (Figure 2c.). The process of destroying organic material was conducted with the use of $20 \mathrm{ml} 0.05 \mathrm{M} \mathrm{Fe}$ (II) and 20\% hydrogen peroxide with a volume of $20 \mathrm{ml}$ to ensure there was no remaining of organic materials. The samples were heated on hotplate with a temperature of $75^{\circ} \mathrm{C}$ for 45 minutes. In every $20 \mathrm{ml}$ of samples were added about $6 \mathrm{~g}$ of $\mathrm{NaCl}$ to separate microplastics from the organic materials (Misura et al., 2015; GESAMP, 2016).

Microplastic samples that have been processed from the WPO process were then moved to a density separator (Figure 2d.). This process aimed to separate the organic materials and the microplastics for an overnight. Floating particles that were considered microplastics were then filtered using $0.33 \mathrm{~mm}$ size and identified with a microscope or identified visually (Misura et al., 2015).

The last step in analyzing microplastics is to identify the acquired microplastics based on their class (fibers, films, fragments or pellets) visually using microscope. Identified microplastics was then placed into a vial and total microplastics weight was calculated (Misura et al., 2015). Microplastics were identified according to the criteria in which fragment cannot be torn apart with tweezer with sharp and broken edges of irregular shapes; fiber is equally thick throughout the entire length and is not tapered at the end; and film is very thin as part of the sheets of plastic bags (Hidalgo-Ruz et al., 2012b; Mohamed Nor and Obbard, 2014; Zobkov and Esiukova, 2017; Dai et al., 2018).

\section{Results and Discussion}

There were three types of microplastic found: fiber (Figure 3a.), film (Figure 3b.) and fragment (Figure 3c.). These results were similar to the result of Kovač Viršek et al. (2016) where microplastic generally found in the aquatic environment were secondary microplastics which originated from cloth 
fiber and fragments of bigger plastic litter. Based on Table 1, it can be observed that fragments were the most abundant type of microplastics in both sampling methods. This was supported with the condition of the Bali Strait where the sampling conducted close to a fishing port and an estuary area where both locations have been contaminated by fishing and domestic wastes. Macroplastics found in the study area were also indicated that the majorities of wastes were from the domestic wastes and fish nets. The total abundance of microplastics obtained by manta net was higher compared to the abundance of microplastics obtained by plankton net (Figure 4.). The total abundance of microplastics from manta net was $32.48 \times 10^{2}$ particles. $\mathrm{m}^{-3}$. On the other hand, the total abundance of microplastic obtained by plankton net was $16.33 \times 10^{2}$ particles. $\mathrm{m}^{-3}$.

The results of this study were similar to the previous studies that use the same methods. Moore et al. (2011) in Los Angeles river found the abundance of microplastics using manta net was $12.9 \times 10^{2}$ particles. $\mathrm{m}^{-3}$. A study on the shores of Kyeonggi Beach-South Korea using a hand net with $20 \mu \mathrm{m}$ mesh (Chae et al., 2015) and another study in Sweden using a hand net $80 \mu \mathrm{m}$ mesh (Nore'n, 2007) showed that the results were nearly identical with this research with a microplastic abundances were in the range of $10-42.27 \times 10^{2}$ particles. $\mathrm{m}^{-3}$ and 1.5- $24 \times 10^{2}$ particles. $\mathrm{m}^{-3}$, respectively.

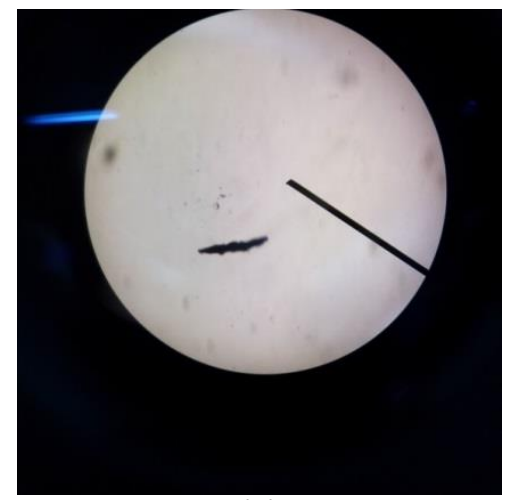

(a)

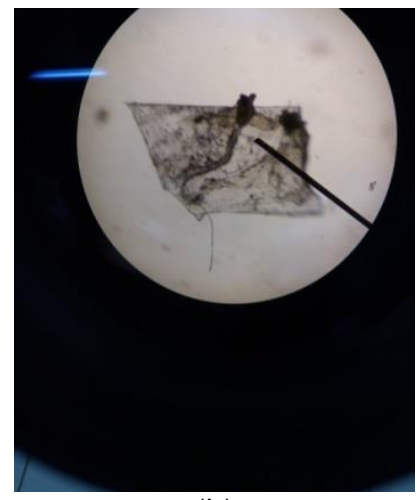

(b)

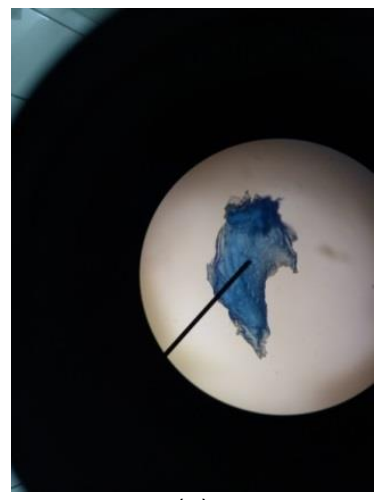

(c)

Figure 3. The types of microplastics found in the study areas: fiber (a), film (b) and fragment (c)

Table 1. Percentage of microplastic types found using both sampling tools in the Bali Strait

\begin{tabular}{ccc}
\hline \multirow{2}{*}{ Microplastic type } & \multicolumn{3}{c}{ Percentage (\%) } \\
\cline { 2 - 3 } & Manta net & Plankton net \\
\hline Fiber & 24.11 & 26.53 \\
Film & 34.04 & 26.53 \\
Fragment & 41.84 & 46.94 \\
\hline
\end{tabular}

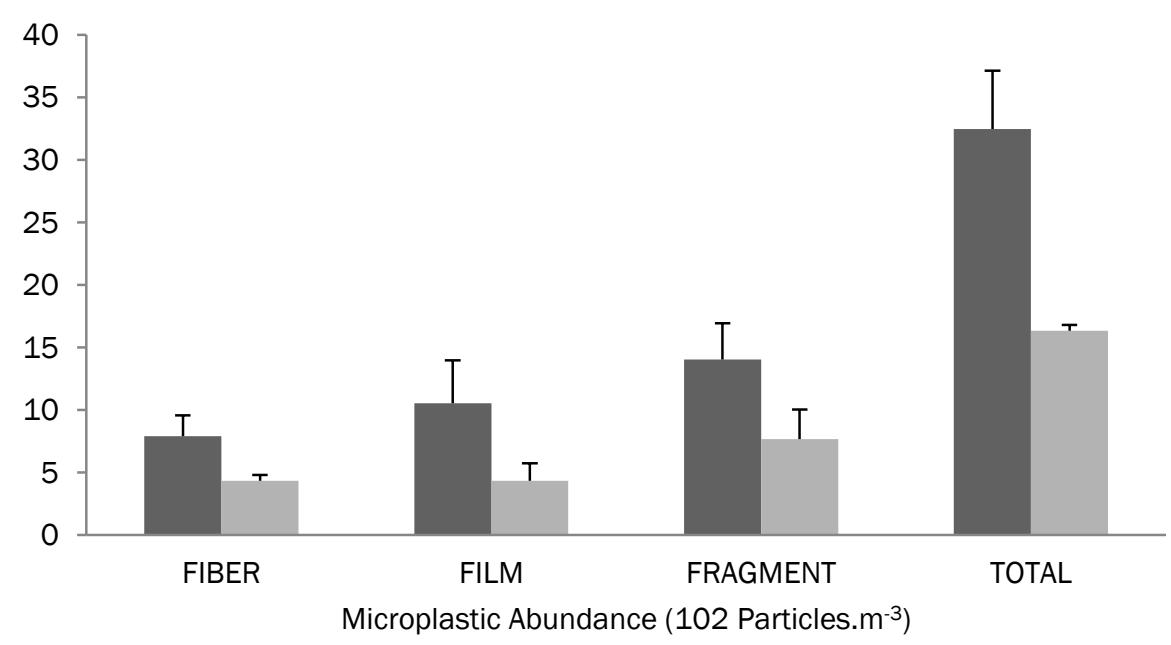

Figure 4. Microplastic abundances in the Bali Strait sampled using manta net (black) and plankton net (grey) 
A significant gap exists between the total abundances of microplastics obtained using manta net and plankton net was likely caused by a few factors; two of which were the mesh size and sampling methods. In the manta net method, water was sampled by pulling the net out by the boat which could increase the chance of microplastics being filtered compared to the plankton net method which was conducted in stationery mode. In addition, the mesh size of the manta net $(250 \mu \mathrm{m})$ was smaller than the mesh size of plankton net $(20 \mu \mathrm{m})$ which may cause microplastics with smaller dimensions to be better filtered and collected (GESAMP, 2016).

The applications of manta net and plankton net in sampling microplastics have its own advantages and disadvantages. The advantage of using manta net is the ability to sample a wider area, thus resulted in the ability to filter a bigger volume of water compared to the plankton net method. Manta net method is considered better to study particles that are smaller than $1 \mathrm{~mm}$ (Setälä et al., 2016). However, the disadvantage of this method is the fact that waves and the increasing speed of the boat will cause the instability of the manta that may lead to the failure of sampling process (Kovač Viršek et al., 2016). On the other hand, wave and also the speed of the boat do not have significant impact on the microplastics sampling using plankton net. In addition, sampling using plankton net is also considered cheaper and more practical compared to the sampling using manta net, although it obtains a smaller number of microplastics.

\section{Conclusion}

There were three types of microplastics found in the Bali Strait: fibers, films and fragments with fragments were the dominant type. Domestic and fisheries activities might contribute to the microplastics pollution in the study area. Higher abundances of microplastics were found using manta net compared to the abundance of microplastics using plankton net. Both sampling tools have its own advantages and disadvantages. According to the results of this study, manta net can be considered as a better tool to study microplastics due to its smaller mesh size and wider coverage of the sampling area.

\section{Acknowledgement}

This research was supported by Institute for Marine Research and Observation (IMRO) Bali who provided insight and expertise that greatly assisted the research sampling and also Marine Research Exploration and Management Research Group
(MEXMA) who helped with the interpretations and conclusions of this paper.

\section{References}

Chae, D.-H., Kim, I.-S., Kim, S.-K., Song, Y.K. \& Shim, W.J., 2015. Abundance and Distribution Characteristics of Microplastics in Surface Seawaters of the Incheon/Kyeonggi Coastal Region. Arch. Environ. Contam. Toxicol. 69, 269-278. doi : 10.1007/s00244-015-0173-4

Coppock, R.L., Cole, M., Lindeque, P.K., Queirós, A.M. \& Galloway, T.S., 2017. A small-scale, portable method for extracting microplastics from marine sediments. Environ. Pollut. 230: 829-837.

Dai, Z., Zhang, H., Zhou, Q., Tian, Y., Chen, T., Tu, C., $\mathrm{Fu}, \quad$ C. \& Luo, Y., 2018. Occurrence of microplastics in the water column and sediment in an inland sea affected by intensive anthropogenic activities. Environ. Pollut. 242: 1557-1565. doi: 10.1016/j.envpol.2018.07.1 31

Gago, J., Filgueiras, A., J. Frias, Pedrotti, M.L., Suaria, G., Tirelli, V., Andrade, J., Nash, R., O'Connor, I., Lopes, C., Caetano, M., Raimundo, J., Carretero, O., Viñas, L., Antunes, J.C., Bessa, F., Sobral, P., Goruppi, A., Aliani, S., Palazzo, L., Lucia, G.A.D., Camedda, A., Muniategui-Lorenzo, S., Grueiro, G., Fernández-González, V. \& Gerdts, G., 2018. Standardised protocol for monitoring microplastics in seawater. JPI-Oceans BASEMAN project. doi: 10.13140/rg.2.2.1418 1.45282

GESAMP, 2016. Source, Fate, and Effects of Microplastics in The Marine Environment: Part Two of A Global Assessment, Report and Studies. International Maritime Organization.

Gregory, M.R., 1996. Plastic 'scrubbers' in hand cleansers: a further (and minor) source for marine pollution identified. Mar. Pollut. Bull. 32: 867-871.

Hastuti, A.R., Yulianda, F. \& Wardiatno, Y., 2014. Spatial distribution of marine debris in mangrove ecosystem of Pantai Indah Kapuk, Jakarta. Bonorowo Wetl. 4: 94-107.

Hidalgo-Ruz, V., Gutow, L., Thompson, R.C. \& Thiel, M., 2012a. Microplastics in the Marine Environment: A Review of the Methods Used for 
Identification and Quantification. Environ. Sci. Technol. 46: 3060-3075. doi: 10.1021/es203 1505

Hidalgo-Ruz, V., Gutow, L., Thompson, R.C. \& Thiel, M., 2012b. Microplastics in the Marine Environment: A Review of the Methods Used for Identification and Quantification. Environ. Sci. Technol. 46: 3060-3075. doi: 10.1021/es203 1505

Kovač Viršek, M., Palatinus, A., Koren, Š., Peterlin, M., Horvat, P. \& Kržan, A., 2016. Protocol for Microplastics Sampling on the Sea Surface and Sample Analysis. J. Vis. Exp., 118: p.e55161. doi : 10.3791/55161

Misura, J., Baker, J., Foster, G. \& Arthur, C., 2015. Laboratory Methods for The Analysis of Microplastics in The Marine Environment: Recommendations for quantifying synthetic particles in waters and sediments. NOAA Technical Memorandum NOS-OR\&R-48.
Mohamed Nor, N.H. \& Obbard, J.P., 2014. Microplastics in Singapore's coastal mangrove ecosystems. Mar. Pollut. Bull. 79: 278-283. doi : 10.1016/j.marpolbul.2013.11.025

Nore 'n, F., 2007. Small Plastic Particles in Coastal Swedish Waters. KIMO Swed.

Setälä, O., Magnusson, K., Lehtiniemi, M., Norén, F., 2016. Distribution and abundance of surface water microlitter in the Baltic Sea: A comparison of two sampling methods. Mar. Pollut. Bull. 110:177-183. doi: 10.1016/j.mar polbul.2016.06. 065

Zobkov, M. \& Esiukova, E., 2017. Microplastics in Baltic bottom sediments: Quantification procedures and first results. Mar. Pollut. Bull. 114: 724-732. doi: 10.1016/j.marpolbul.201 6.10 .060 